\title{
ZERO SPAN IS A SEQUENTIAL STRONG WHITNEY-REVERSIBLE PROPERTY
}

\author{
AKIRA KOYAMA \\ (Communicated by Doug W. Curtis) \\ Dedicated to Professor Jun-ichi Nagata on his 60th birthday
}

\begin{abstract}
The concept of span of metric spaces was introduced by Lelek [5]. Span is an important concept in regard to chainability of continua. In this paper, motivated by recent results $[\mathbf{2}, \mathbf{1 1}]$, we show that zero span is a sequential strong Whitney-reversible property.
\end{abstract}

1. Introduction. All spaces are assumed to be nonempty metric spaces, and all maps to be continuous functions. A continuum is a compact connected space and a Peano continuum is a locally connected continuum. The hyperspace of a continuum $X$ is the space

$$
C(X)=\{A \subseteq X \mid A \text { is nonempty, compact and connected }\}
$$

metrized with the Hausordff metric [9]. Let $F_{1}(X)=\{\{x\} \mid x \in X\}$. A Whitney map for $C(X)$ is a map $\mu: C(X) \rightarrow[0, \infty)$ such that

(i) $\mu(\{x\})=0$ for each $x \in x$, and

(ii) if $A, B \in C(X)$ and $A \subsetneq B$, then $\mu(A)<\mu(B)$.

Namely, a Whitney map is a size function, in some sense. For a Whitney map $\mu$ for $C(X)$, Whitney levels $\mu^{-1}(t), 0 \leq t \leq \mu(X)$ are coverings of $X$ which, as $t$ gets to close to zero, converge to $\mu^{-1}(0)=F_{1}(X) \cong X$. Therefore, it is interesting to obtain information about the structure of Whitney levels, and to determine those properties which are preserved by the convergence of positive Whitney levels $\mu^{-1}(t), 0<t \leq \mu(X)$, to the zero level. A topological property $P$ is said to be sequentially strongly Whitney-reversible (resp., strongly Whitney-reversible) [9, 10], provided that if a continuum $X$ has a Whitney map $\mu$ for $C(X)$ such that there exists a decreasing sequence $\left\{t_{n}\right\}_{n \geq 1}$ in $(0, \mu(X)]$ such that $\lim _{n} t_{n}=0$ and each $\mu^{-1}\left(t_{n}\right), n \geq 1$, has property $P$ (resp., $\mu^{-1}(t)$ has property $P$ for every $0<t<\mu(X))$, then $X$ has property $P$. Clearly, every sequential strong Whitneyreversible property is a strong Whitney-reversible property. In $[\mathbf{2}, \mathbf{4}, 9$ and 10], a number of properties were shown to be (sequentially) strongly Whitney-reversible, e.g., chainability, tree-likeness, acyclicity, $\operatorname{dim} \leq n$, being atriodic, etc. In fact, it is

Received by the editors July 28,1986 .

1980 Mathematics Subject Classification (1985 Revision). Primary 54B20, 54F20; Secondary $54 \mathrm{~F} 65$.

Key words and phrases. Span, surjective span, $\varepsilon$-uniformization, hypersurface, Whitney map, Whitney-reversible property.

This research was done while the author was at the Institute of Mathematics, University of Wroclaw, as a participant in an exchange program by the Japan Society for the Promotion of Science and the Polish Academy of Sciences. 
interesting and important to determine whether or not a given topological property is (sequentially) strongly Whitney-reversible.

The purpose of this paper is to show that zero span is a sequential strong Whitney-reversible property.

2. Definitions and preliminaries. Let $X$ be a connected space with a metric d, and let $\pi_{i}: X \times X \rightarrow X$ denote the $i$ th coordinate projection for $i=1,2$. The surjective span $\sigma^{*}(X)$ (resp., surjective semispan $\sigma_{0}^{*}(X)$ ) of $X$ is the least upper bound of the set of all real numbers $\alpha$ which satisfy the following condition:

There exists a connected subset $C \subseteq X \times X$ such that $\pi_{1}(C)=$ $\pi_{2}(C)=X\left(\right.$ resp.,$\left.\pi_{1}(C)=X\right)$ and $\mathbf{d}(x, y) \geq \alpha$ for each $(x, y) \in C$.

The span $\sigma(X)$ and the semispan $\sigma_{0}(X)$ of $X$, not necessarily connected, are

$$
\begin{aligned}
\sigma(X) & =\sup \left\{\sigma^{*}(A) \mid A \subseteq X, A \neq \varnothing \text { and connected }\right\}, \text { and } \\
\sigma_{0}(X) & =\sup \left\{\sigma_{0}^{*}(A) \mid A \subseteq X, A \neq \varnothing \text { and connected }\right\} .
\end{aligned}
$$

It is known that every chainable continuum has zero span (see [5]). Recently, Oversteegen and Tymchatyn [11] characterized continua $X$ with $\sigma^{*}(X)=0$ in terms of uniformizations of two sequences of arcs converging in $C(X)$ to $X$. In this paper, we will essentially use their characterization. Let $I$ be the closed unit interval $[0,1]$. For maps $f, g: I \rightarrow X$ and a positive number $\varepsilon>0$, we say that $g$ can be $\varepsilon$-uniformized with a piece of $f$ if there exist maps $a, b: I \rightarrow I$ such that $b(I)=I$ and $\mathbf{d}(f \circ a(t), g \circ b(t))<\varepsilon$ for each $t \in I$.

LEMMA 1 [11, THEOREM 4]. (1) Let $X \subseteq Y$ be continua and let $\varepsilon>0$ be a positive number. If $\sigma^{*}(X)<\varepsilon$, then the following condition $(*)_{\varepsilon}$ is satisfied:

For each pair of sequences $\left\{f_{i}\right\}_{i \geq 1},\left\{g_{i}\right\}_{i \geq 1}$ of maps $f_{i}, g_{i}: I \rightarrow Y$ $(*)_{\varepsilon} \quad$ such that $\operatorname{Lim}_{i} f_{i}(I)=\operatorname{Lim}_{i} g_{i}(I)=X$, there exists an integer $n \geq 1$ such that for each $i \geq n$, either $f_{i}$ is $\varepsilon$-uniformized with a piece of $g_{i}$ or $g_{i}$ is $\varepsilon$-uniformized with a piece of $f_{i}$.

(2) Let $X$ be a continuum in a Peano continuum $Y$. If for each positive number $\varepsilon>0$, the condition $(*)_{\varepsilon}$ is satisfied, then $\sigma^{*}(X)=0$.

Some continuity property of spans is useful. The following is obtained by a slight modification of the proof of [7, 3.1].

LEMMA 2. Let $X \subseteq Y$ be continua and let $\varepsilon>0$ be a positive number. If there exists a sequence $\left\{X_{n}\right\}_{n \geq 1}$ of continua in $Y$ such that $\operatorname{Lim}_{n} X_{n}=X$ and $\tau\left(X_{n}\right) \geq \varepsilon$ for each $n \geq 1$, then $\tau(\bar{X}) \geq \varepsilon$, where $\tau=\sigma^{*}, \sigma_{0}^{*}, \sigma$, or $\sigma_{0}$.

Basic facts about Whitney maps and Whitney levels may be found in [9].

3. Main results. In this section we will show that zero span is a sequential strong Whitney-reversible property. In fact, we will obtain a more general result, as follows:

THEOREM. Let $X$ be a continuum and let $\mu$ be any Whitney map for $C(X)$. If there exists a decreasing sequence $\left\{t_{n}\right\}_{n \geq 1}$ in $(0, \mu(X)]$ such that $\lim _{n} t_{n}=t$ and $\sigma\left(\mu^{-1}\left(t_{n}\right)\right)=0$ for each $n \geq 1$, then $\sigma\left(\mu^{-1}(t)\right)=0$.

PROOF. Since zero span is a topological invariant in the class of continua, we may assume that $X$ is a subcontinuum of the Hilbert cube $\mathbf{Q}$ with a metric $\mathbf{d}$. By 
[12], there exists a Whitney map $\tilde{\mu}$ for $C(\mathbf{Q})$ such that $\tilde{\mu} \mid C(X)=\mu$. Then $\tilde{\mu}^{-1}(t)$ is a Peano continuum. Hence, applying Lemma 1, we will show that, for every nonempty subcontinuum $\Lambda$ of $\mu^{-1}(t), \sigma^{*}(\Lambda)=0$.

Let $\left\{f_{i}\right\}_{i \geq 1},\left\{g_{i}\right\}_{i \geq 1}$ be a given pair of sequences of maps $f_{i}, g_{i}: I \rightarrow \tilde{\mu}^{-1}(t)$ such that $\operatorname{Lim}_{i} f_{i}(I)=\operatorname{Lim}_{i} g_{i}(I)=\Lambda$ and let $\varepsilon>0$ be a positive number. By [3], there exists a positive number $\eta>0$ such that

$$
\text { if } A, B \in C(\mathbf{Q}), A \subseteq B \text {, and } \tilde{\mu}(B)-\tilde{\mu}(A)<\eta \text {, then } \mathbf{d}_{H}(A, B)<\varepsilon / 3 \text {, }
$$

where $\mathbf{d}_{H}$ is the Hausdorff metric on $C(\mathbf{Q})$ induced by the metric $\mathbf{d}$. Since $\lim _{n} t_{n}=$ $t$, there exists an integer $n \geq 1$ such that $t_{n}-t<\eta$. Then

$$
\text { if } A \in \tilde{\mu}^{-1}(t), B \in \tilde{\mu}^{-1}\left(t_{n}\right) \text {, and } A \subseteq B \text {, then } \mathbf{d}_{H}(A, B)<\varepsilon / 3 \text {. }
$$

Let take a decreasing sequence $\left\{X_{k}\right\}_{k \geq 1}$ of Peano continua in $\mathbf{Q}$ such that for each $k \geq 1, X_{k}$ is a closed neighborhood of $X_{k+1}$ in $\mathbf{Q}$ and $\bigcap_{k \geq 1} X_{k}=X$. Then we can easily see that

$$
\operatorname{int}_{\tilde{\mu}^{-1}\left(t_{n}\right)}\left(\tilde{\mu}^{-1}\left(t_{n}\right) \cap C\left(X_{k}\right)\right) \supseteq \tilde{\mu}^{-1}\left(t_{n}\right) \cap C\left(X_{k+1}\right) \quad \text { for each } k \geq 1,
$$

and

$$
\bigcap_{k \geq 1}\left(\tilde{\mu}^{-1}\left(t_{n}\right) \cap C\left(X_{k}\right)\right)=\mu^{-1}\left(t_{n}\right) .
$$

Since $\sigma\left(\mu^{-1}\left(t_{n}\right)\right)=0$, by (2), (3), and Lemma 2, there exists an integer $k \geq 1$ such that

$$
\sigma\left(\tilde{\mu}^{-1}\left(t_{n}\right) \cap C\left(X_{k}\right)\right)<\varepsilon / 3 .
$$

Then by (2) and (3), passing to subsequences if necessary, we may assume that $f_{i}(I), g_{i}(I) \subseteq \tilde{\mu}^{-1}\left(t_{n}\right) \cap C\left(X_{k}\right)$ for each $i \geq 1$.

Since $X_{k}$ is a Peano continuum, $X_{k}$ admits a convex metric $\rho$. Then a function $K: C\left(X_{k}\right) \times[0, \infty) \rightarrow C\left(X_{k}\right)$ given by

$$
K(A, s)=\left\{x \in X_{k} \mid \rho(x, a) \leq s \text { for some } a \in A\right\} \quad \text { for } A \in C\left(X_{k}\right) \text { and } s \in[0, \infty)
$$

is continuous (see [8]). Hence we have the map $h: \tilde{\mu}^{-1}(t) \cap C\left(X_{k}\right) \rightarrow \tilde{\mu}^{-1}\left(t_{n}\right) \cap C\left(X_{k}\right)$ defined by, for each $A \in \tilde{\mu}^{-1}(t) \cap C\left(X_{k}\right)$,

$$
h(A)=K\left(A, \alpha\left(A, t_{n}\right)\right), \quad \text { where } \tilde{\mu}\left(K\left(A, \alpha\left(A, t_{n}\right)\right)\right)=t_{n}
$$

(see [2 and 10]).

Let consider the pair of sequences $\left\{f_{i}^{*}\right\}_{i \geq 1},\left\{g_{i}^{*}\right\}_{i \geq 1}$ of maps $f_{i}^{*}=h \circ f_{i}, g_{i}^{*}=$ $h \circ g_{i}: I \rightarrow \tilde{\mu}^{-1}\left(t_{n}\right) \cap C\left(X_{k}\right)$. Then $\operatorname{Lim}_{i} f_{i}^{*}(I)=\operatorname{Lim}_{i} g_{i}^{*}(I)=h(\Lambda)$. By $(4)$, $\sigma^{*}(h(\Lambda)) \leq \sigma\left(\tilde{\mu}^{-1}\left(t_{n}\right) \cap C\left(X_{k}\right)\right)<\varepsilon / 3$. Hence by Lemma $1(1)$, there exists an integer $i_{0} \geq 1$ such that

for each $i \geq i_{0}$, either $f_{i}^{*}$ is $\varepsilon / 3$-uniformized with a piece of $g_{i}^{*}$ or $g_{i}^{*}$ is $\varepsilon / 3$-uniformized with a piece of $f_{i}^{*}$.

For an arbitrary integer $i \geq i_{0}$, assume that $f_{i}^{*}$ is $\varepsilon / 3$-uniformized with a piece of $g_{i}^{*}$. Thus, there exists maps $a_{i}, b_{i}: I \rightarrow I$ such that

$$
a_{i}(I)=I
$$


and

$$
\mathbf{d}_{H}\left(f_{i}^{*} \circ a_{i}(s), g_{i}^{*} \circ b_{i}(s)\right)<\varepsilon / 3 \text { for each } s \in I .
$$

For each $s \in I, f_{i} \circ a_{i}(s) \in \tilde{\mu}^{-1}(t)$, and by (5),

$$
f_{i} \circ a_{i}(s) \subseteq h\left(f_{i} \circ a_{i}(s)\right)=f_{i}^{*} \circ a_{i}(s) \in \tilde{\mu}^{-1}\left(t_{n}\right) .
$$

Hence, by (1),

$$
\mathbf{d}_{H}\left(f_{i} \circ a_{i}(s), f_{i}^{*} \circ a_{i}(s)\right)<\varepsilon / 3 \quad \text { for each } s \in I .
$$

Similarly, we can see that

$$
\mathbf{d}_{H}\left(g_{i} \circ b_{i}(s), g_{i}^{*} \circ b_{i}(s)\right)<\varepsilon / 3 \quad \text { for each } s \in I .
$$

Hence, by (7), (8) and (9),

$$
\begin{aligned}
\mathbf{d}_{H}\left(f_{i} \circ a_{i}(s), g_{i} \circ b_{i}(s)\right) \leq & \mathbf{d}_{H}\left(f_{i} \circ a_{i}(s), f_{i}^{*} \circ a_{i}(s)\right) \\
& +\mathbf{d}_{H}\left(f_{i}^{*} \circ a_{i}(s), g_{i}^{*} \circ b_{i}(s)\right) \\
& +\mathbf{d}_{H}\left(g_{i}^{*} \circ b_{i}(s), g_{i} \circ b_{i}(s)\right) \\
< & \varepsilon / 3+\varepsilon / 3+\varepsilon / 3 \\
= & \varepsilon \text { for each } s \in I .
\end{aligned}
$$

It follows from (6) and (10) that $f_{i}$ is $\varepsilon$-uniformized with a piece of $g_{i}$.

Similarly, we can see that for $i \geq i_{0}$, if $g_{i}^{*}$ is $\varepsilon / 3$-uniformized with a piece of $f_{i}^{*}$, then $g_{i}$ is $\varepsilon$-uniformized with a piece of $f_{i}$. Therefore, for each $i \geq i_{0}$, either $f_{i}$ is $\varepsilon$-uniformized with a piece of $g_{i}$ or $g_{i}$ is $\varepsilon$-uniformized with a piece of $f_{i}$. From Lemma $1(2)$, we have that $\sigma^{*}(\Lambda)=0$. It follows that $\sigma\left(\mu^{-1}(t)\right)=\sup \left\{\sigma^{*} \in \Lambda\right) \mid \Lambda \in$ $\left.C\left(\mu^{-1}(t)\right)\right\}=0$.

\section{COROLLARY 1. Zero span is a sequential strong Whitney-reversible property.}

By [11], continua with zero surjective semispan are weakly chainable, atriodic and tree-like. Hence we have

COROLlaRY 2. Let $X$ be a continuum. If there exist a Whitney map $\mu$ for $C(X)$ and a decreasing sequence $\left\{t_{n}\right\}_{n \geq 1}$ in $(0, \mu(X)]$ such that $\lim _{n} t_{n}=0$ and $\sigma\left(\mu^{-1}\left(t_{n}\right)\right)=0$ for each $n \geq 1$, then $X$ is weakly chainable, atriodic, and tree-like.

By [1], we know that for a continuum $X, \sigma(X)=0$ if and only if $\sigma_{0}(X)=$ 0 . Hence zero semispan is also a sequential strong Whitney-reversible property. However, in the proof of the Theorem, the map $h$ need not be surjective. Therefore we do not know whether or not zero surjective (semi)span is a sequential strong Whitney-reversible property.

\section{REFERENCES}

1. J. F. Davis, The equivalence of zero span and zero semispan, Proc. Amer. Math. Soc. 98 (1984), 133-138.

2. H. Kato, Shape properties of Whitney maps for hyperspaces, Trans. Amer. Math. Soc. 297 (1986), 529-546.

3. J. L. Kelley, Hyperspaces of continua, Trans. Amer. Math. Soc. 52 (1942), 22-36.

4. A. Koyama, A note on some strong Whitney-reversible properties, Tsukuba J. Math. 4 (1980), 313-316. 
5. A. Lelek, Disjoint mappings and the span of spaces, Fund. Math. 55 (1964), 199-214.

6. $\ldots$, On surjective span and semispan of connected metric spaces, Colloq. Math. 37 (1977), 35-45.

7 , The span and the width of continua, Fund. Math. 98 (1978), 181-199.

8 S. B. Nadler, Jr., A characterization of locally connected continua by hyperspace retraction, Proc. Amer. Math. Soc. 67 (1977), 167-176.

9. __ Hyperspaces of sets, Pure and Appl. Math., vol. 49, Marcel Dekker, New York, 1978.

10. _ Whitney-reversible properties, Fund. Math. 109 (1980), 235-248.

11. Lex G. Oversteegen and E. D. Tymchatyn, On span and weakly chainable continua, Fund. Math. 122 (1984), 159-174.

12. J. E. Ward, Jr., Extending Whitney maps, Pacific J. Math. 93 (1981), 465-469.

Institute of Mathematics, University of Wroclaw, Pl. GRunWaldzki 2/4, 50-384, WROCLAW, POLAND

Department of Mathematics, OSAKa Kyoiku UniVersity, IKeda-City, OSAKa, 563 , JAPAN 